\title{
Equilibrium Decision Method for Earthquake First-Aid Medicine Allocation Based on Demand Information Updating
}

\author{
Yong Ye, ${ }^{1}$ Shalei Zhan, ${ }^{2}$ and Shih-Yung Wei ${ }^{3}$ \\ ${ }^{1}$ College of Humanities and Management, Wenzhou Medical University, Wenzhou 325035, China \\ ${ }^{2}$ School of Computer Science and Information Engineering, Zhejiang Gongshang University, Hangzhou 310058, China \\ ${ }^{3}$ Academy of Finance Research, Wenzhou University, Wenzhou 325035, China \\ Correspondence should be addressed to Shih-Yung Wei; noahsywei@163.com
}

Received 3 February 2016; Revised 22 July 2016; Accepted 1 August 2016; Published 31 January 2017

Academic Editor: Francesco Franco

Copyright (C) 2017 Yong Ye et al. This is an open access article distributed under the Creative Commons Attribution License, which permits unrestricted use, distribution, and reproduction in any medium, provided the original work is properly cited.

\begin{abstract}
The allocation of rescue resources after an earthquake has become a popular research topic in the field of emergency management. The allocation of first-aid medicine for earthquake rescue has stronger time sensitivity than that of general rescue materials. This study focuses on the problem of first-aid medicine allocation in earthquake response. First, we consider the incompleteness and renewal of decision information in an emergency environment, as well as the balance between the risk of decision error and delay. Second, we propose an equilibrium decision method for the allocation of first-aid medicine in earthquake rescue based on information update. This method attempts to realize a fair allocation to all disaster places and minimize total transport time loss. Third, a simulation analysis is performed in which the proposed method is applied to the first-aid medicine allocation problem in the Wenchuan earthquake response. Results show that the method can be used to create a good allocation plan in an earthquake rescue situation.
\end{abstract}

\section{Introduction}

Earth has been subjected to earthquakes during its entire geological period. Historically, the frequent occurrence of catastrophic or major earthquakes has resulted in a considerable loss of life and property worldwide. The magnitude 8.3 earthquake that hit San Francisco on April 18, 1906, took more than 60,000 lives. The great Kanto earthquake that occurred in Japan on September 1, 1923, destroyed 600,000 buildings, took 143,000 lives, and left 200,000 injured and 500,000 homeless. The terrible fire that occurred in the business district of Tokyo at the same time took 240,000 lives in Yokohama Park. Earthquakes also trigger landslides, debris flow, and other secondary disasters, all of which cause large numbers of casualties. A magnitude 8.9 earthquake with an epicentral intensity of more than 11 hit Chile on May 22, 1980, and hundreds of stronger earthquakes followed continuously. Among the earthquakes that followed, 3 were more than 8.0 in magnitude and 10 were more than 7.0. The great Kanto earthquake also triggered a violent tsunami and a severe volcanic eruption, taking 5700 lives. A magnitude
8.1 earthquake occurred in the Pacific Ocean, off the coast of the Mexican state, on September 19, 1985. A magnitude 7.5 aftershock occurred the next day, taking more than 7000 lives, causing injury to 11,000 people, and leaving more than 0.3 million homeless; the economic loss totaled USD 1.1 billion. The magnitude 7.2 Great Hanshin earthquake occurred on January 17, 1995, taking 5466 lives and leaving more than 30,000 injured and hundreds of thousands of others homeless; the victims reached more than 1.4 million. A magnitude 7.4 earthquake occurred in the western part of Turkey on August 17, 1999, taking more than 13,000 lives. A magnitude 8.7 earthquake occurred on December 26, 2004 , and a tsunami was triggered off the northwest coast of Sumatra, Indonesia, killing 188,149 people in the countries around the Indian Ocean and with 43,861 still missing. A magnitude 7.8 earthquake occurred in Pakistan on October 8, 2005, affecting Pakistan, India, Afghanistan, and Indonesia. More than 79,000 Pakistanis lost their lives, over 60,000 were injured, and 3.3 million were left homeless. A magnitude 7.4 earthquake occurred in Haiti on January 12, 2010, taking 222,500 lives and injuring 196,000 people. A magnitude 9.0 
earthquake occurred in Japan on March 11, 2011, and triggered a tsunami, causing unit numbers 1-4 of the Fukushima I Nuclear Power Plant to develop a nuclear leak, taking 13,498 lives, and leaving 14,734 people still missing. Clearly, global earthquake relief work is arduous.

Unlike other disasters, earthquakes are characterized by strong suddenness, huge destructiveness, and high defense difficulty. After an earthquake, the demands for drugs, food, tents, and other supplies in disaster areas increase sharply within a short period. The construction of emergency resources in China has many weak links, and the demands of disaster emergency cannot be fully met. For example, a shortage of emergency rescue materials occurred during the Wenchuan (WC), Yushu, and Ludian earthquakes. The shortage or delay of emergency medical supplies, particularly for sterilization, anti-infection, anesthesia, and stanch bleeding, prevents the timely treatment of victims. For example, numerous victims died in Indonesia earthquake because of drug shortage.

In medical rescue, treatment during the first three days after an earthquake is critical; the effect of medical rescue will gradually weaken after this period [1]. Drug allocation for earthquake first aid has higher time sensitivity than that for general rescue supplies. On the one hand, the acquired decision-making information is insufficient if a decision is made instantly, and thus decision-making efficiency may be low. This phenomenon can reduce the urgency of decisionmaking time in the late stage, resulting in invalid execution and the complexity of problems. Thus, the risk of decision error is high and considerable manpower and material resources are consumed. On the other hand, the acquired decision-making information is more robust if a decision is made later, and thus decision-making efficiency may be better. This situation can reduce the risk of decision error but may delay decision making because of the further deterioration of the disaster, thereby increasing the risk of delayed implementation. An ideal allocation plan can be obtained once a simple program is designed if complete information on the demand for rescue drugs, resource supply, and transportation is obtained. However, the occurrence and development of earthquake disaster are accompanied by numerous uncertainties, and acquiring complete disaster information is difficult. Therefore, a decision maker needs to utilize the available information, which includes sample information obtained at the current stage and historical information of related disasters, to make a scientific and reasonable allocation plan. The sample information at the current stage can be continuously updated through observation, that is, information renewal of drug allocation for earthquake rescue. Multiple vectors are used to describe the related information in the decision-making process of emergency resource allocation for earthquake disaster, namely, group information. The group information for the allocation decision of rescue drugs is continuously acquired and updated with the constant disaster evolution, and the related allocation decision is dynamically optimized.

Therefore, a decision maker should consider several problems in the decision-making process, including when to allocate, where to allocate, how much to allocate, and how to allocate. He or she needs to focus on the balance between the decision time and risk of rescue medicine allocation under an uncertain environment and the dynamic renewal of demand information. Based on the summary of results of previous studies and using Bayesian analysis theory, this study comprehensively considers the balance of risks of decision error and delay and constructs a decisionmaking method for rescue medicine allocation under the renewal of demand information. This method is applied to an example of rescue medicine allocation for the WC earthquake to perform a simulation analysis. The result shows that an emergency decision maker can update data by inputting the corresponding demand information, acquiring the optimal decision time under the balance between decision time and risk of rescue medicine allocation, and creating an allocation plan with the minimum transport time under a fair allocation principle.

\section{Research Status}

The following paragraphs summarize the related studies on the supply and demand for earthquake first-aid medicine and decision-making method for the allocation of disaster rescue supplies.

2.1. Study on the Supply and Demand for Earthquake FirstAid Medicine. Studies on emergency medicine have achieved fruitful results, mainly by focusing on the development of emergency medicine, emergency medical service, and analysis of all types of common diseases after a disaster [2]. At present, studies on the supply and demand for earthquake first-aid medicine utilize the practical data of earthquake medical rescue to analyze the main injury types caused by earthquake disasters. Moreover, these studies use the supply, demand, and application of related drugs to raise suggestions on enhancing the reservation, management, and application of earthquake first-aid drugs.

Studies on the supply of earthquake first-aid drugs mainly include the supply characteristics, supply security, donation management of rescue drugs, production purchasing, dispatching, transportation, and existing problems. Donation is the major source of earthquake first-aid drugs. However, some donation drugs are expired, of poor quality, or useless; thus, the use efficiency of drugs is low. A total of 5000 tons of drugs and medical tools were received in the rescue process of the San Francisco earthquake in 1989, but only approximately $30 \%$ could be used immediately [3]. Xiao et al. [4] indicated that the dispatch, distribution, donation rules, and use management of rescue drugs in China need to be standardized further. Mori et al. [5] also analyzed the significant effect of drug shortage on the rescue during the large earthquake in Japan in 2011.

Studies on the demand for earthquake first-aid drugs according to historical earthquake first-aid practices have analyzed the demand for earthquake first-aid drugs on the basis of the studies on earthquake injury and medication. Fracture, soft tissue injury, and crush syndrome are the most significant factors in earthquake-disaster-caused casualties [6]. Kazancioglu et al. [7] analyzed the change in the demand 
for blood of crush syndrome patients by using first-aid data from the Marmara earthquake. Their result showed that the demand for blood was highest during the first week after the earthquake. Kudo et al. [8] investigated the reservation and demand for drugs, medical tools, and other medical supplies of 14 main hospitals after the East Japan earthquake. Their result showed that the demands of all hospitals for medicine were accommodated on the fourth day after the earthquake.

2.2. Study on the Decision-Making Method for the Allocation of Disaster Rescue Supplies. The allocation of disaster rescue supplies is the main content of an emergency logistics system operation. Existing related studies are mainly focused on solving emergency logistics, transshipment center location, and distribution route selection in an uncertain environment through the model construction and design of solving algorithms.

Studies on the decision-making model of the allocation of disaster rescue supplies have constructed an allocation and dispatch model to satisfy the requirements of a disaster area maximally. Meanwhile, efficiency and fairness have been considered under the conditions of sharply increasing demand, uncertain supply, and road damage by stochastic programming, robust optimization, Bayesian analysis, spacetime network, mixed integer programming, and other theories. Sheu [9] constructed an allocation model of emergency supplies with the maximization of survivor fitness as the objective by starting from the demand and using stochastic programming theory. Haghani et al. [10] established a timespace network model for selecting the distribution route of disaster rescue supplies. Özdamar and Yi [11] established a mixed integer programming model based on emergency logistics combined with crowd evacuation, resource allocation, and route selection.

Studies on the solving algorithm of the allocation model of disaster rescue supplies have considered that the allocation of disaster rescue supplies is a nondeterministic polynomial time-hard problem that involves various factors, such as multiple objectives, starting points, demand points, transportation modes, materials, and agents. The solving algorithms of related decision-making models include Lagrangian relaxation technique, genetic algorithm, bionic algorithm, ant colony optimization, and particle swarm optimization. Chang et al. [12] introduced a multiobjective genetic algorithm based on greedy search to solve a largescale emergency resource allocation model. Zhang et al. [13] designed a bionic algorithm against emergency logistic network route programming problems. Yuan and Wang [14] solved a multiobjective emergency logistics route selection model by using ant colony optimization.

Studies on the supply and demand for earthquake firstaid medicines and the allocation of disaster rescue supplies are relatively abundant. By contrast, studies on the supply and demand for an adaptation strategy of the allocation of rescue medicines are limited. In particular, no study has evaluated the equilibrium decision method between decision time for rescue medicine allocation and risk of decision error from the perspective of information updating in an uncertain environment. In previous studies, a particular algorithm was required to design the solutions to a model because of the model complexity. The solving efficiency was not high, and only a satisfactory solution could be obtained. In consideration of the urgency in an emergency environment, the related model can not only increase the solving efficiency, but also obtain the optimum solution if it is solved using a mature mathematical software. On the basis of the preceding analysis, this study integrates an information updating method with the balanced allocation of earthquake first-aid medicine using Bayesian analysis theory to achieve the balance between the risk of decision error and risk of decision delay while staying true to the principle of fairness. The study likewise aims to use a decision method for earthquake first-aid medicine allocation, which is solved using mature mathematical software.

\section{Equilibrium Decision-Making Method without Information Updating}

3.1. Problems and Symbols. Many disaster points have required emergency rescue medicine supplies after the 2011 East Japan earthquake and other major earthquake disasters. However, local emergency reserve drugs are difficult to maintain during such a sudden increase in demands. Therefore, we need to supply related drugs from other supply points outside the disaster areas. Supposing that $m$ supply points and $n$ disaster points exist, the other parameters are shown in Notations: (i) Symbols.

\subsection{Hypotheses and Definitions}

Hypothesis 1. Compared with life-type emergency rescue supplies, rescue medicine is characterized by a relatively small demand despite its importance. The distribution of rescue medicine can be prioritized if necessary. Therefore, this study assumes that the supply point for rescue medicine has an adequate distribution capability. Hence, transport capacity is not considered in the model.

Hypothesis 2. The requirement of point $j$ in $t$ can be expressed by the population transfer rate. The requirement is denoted by the stochastic variable $\theta_{j t}$ and submits to the detailed distribution. The probability density function is $\pi\left(\theta_{j t}\right)$.

Hypothesis 3. The supply in the supply point is confirmed.

Definition 1. The requirement of point $j$ in $t$ is the function of population transfer rate $\theta_{j t}$, which is defined as follows:

$$
d_{j t}=d\left(\theta_{j t}\right) \text {. }
$$

Definition 2. The loss of decision error $R_{t}^{\text {Error }}$ in $t$ is a function related to the prediction accuracy $p_{t}$, which is defined as follows:

$$
R_{t}^{\text {Error }}=1-p(t)
$$

where $p(t)$ is a monotone increasing function in decision time $t$. 
Definition 3. The loss of decision delay $R_{t}^{\text {Delay }}$ in $t$ is a monotone increasing function related to decision time, which is defined as follows:

$$
R_{t}^{\text {Delay }}=R(t)
$$

Definition 4. The loss of transport time $L_{t}^{T}$ in $t$ is the total time of medicine distribution from each supply point to each disaster point, which is defined as follows:

$$
L_{t}^{T}=\sum_{i=1}^{m} \sum_{j=1}^{n}\left(t_{i j}^{0} \cdot s_{i j t}\right) .
$$

Definition 5. The fair allocation principle ensures that the expected demand satisfaction rates of all disaster points are equal. Thus, the expected demand satisfaction rate of point $j$ in $t$ is defined as follows:

$$
u_{j t}=\frac{\min \left\{\sum_{j=1}^{n} E\left(d_{j t}\right), \sum_{i=1}^{m} S_{i t}\right\}}{\sum_{j=1}^{n} E\left(d_{j t}\right)} .
$$

In (5), when $\sum_{j=1}^{n} E\left(d_{j t}\right)>\sum_{i=1}^{m} S_{i t}$, the expected demand satisfaction rate $u_{j t}$ is less than 1 because the supply is not enough. However, when $\sum_{j=1}^{n} E\left(d_{j t}\right) \leq \sum_{i=1}^{m} S_{i t}$, the expected demand satisfaction rate $u_{j t}$ equals 1 , which means that the supply is enough to satisfy all the demands.

3.3. Decision-Making Model. According to the above hypotheses and definitions, an equilibrium configuration model of earthquake first-aid medicine can be established in an uncertain environment. The specific objectives are as follows: (I) balanced risks of decision error and delay, (II) similar expected demand satisfaction rates of all disaster points, and (III) smallest total loss of transportation time. The model can be established as follows:

$$
\min z=\sum_{i=1}^{m} \sum_{j=1}^{n}\left(t_{i j}^{0} \cdot s_{i j t}\right) .
$$

Thus,

$$
\begin{aligned}
u_{j^{\prime} t} & =u_{j^{\prime \prime} t}, \quad \forall j^{\prime}, j^{\prime \prime} \in J, \\
\sum_{j=1}^{n} s_{i j t} & =S_{i t}, \quad \forall i \in I, \\
\left(R_{t}^{\text {Error }}-R_{t}^{\text {Delay }}\right)^{2} & \leq \alpha, \\
0 & \leq t \leq T_{0}, \\
s_{i j t} & \geq 0 .
\end{aligned}
$$

Equation (6) refers to the objective function; constraint condition (7) indicates that the expected demand satisfaction rates of all disaster points are the same; constraint condition (8) meets the total supply limit of all supply points; constraint condition (9) refers to the equilibrium condition between the risks of decision error and delay; that is, the square of the difference between both is not more than $\alpha$; and constraint condition (10) refers to the range of the variable. The model can be solved using Lingo software.

\section{Equilibrium Decision-Making Method under Demand Information Updating}

4.1. Symbols and Hypotheses. The decision of earthquake first-aid medicine allocation cannot be in place in just one step but is continuously updated with the development of an earthquake disaster. Such a decision is a dynamic allocation based on the related information updates. The balanced allocation of earthquake first-aid medicine based on the demand information update can be modeled using Bayesian analysis [15], which is used to establish related models. The symbols are presented in Notations: (ii) New Symbols.

In an earthquake first-aid medicine allocation model under demand information updating, a decision maker can adopt various rescue medicine allocation plan sets $\mathscr{A}=\{\mathbf{a}\}$ (the plan mainly refers to the quantity of medicine distributed from all supply points to all disaster points) to describe various possible status sets of population transfer rate $\Theta=$ $\{\boldsymbol{\theta}\}$. The decision-making rule $\boldsymbol{\delta}(\mathbf{x})$ shows the action taken $[\boldsymbol{\delta}(\mathbf{x})$ refers to the function from $\mathscr{X}$ to $\mathscr{A}]$ and $\mathbf{X}=\mathbf{x}$ is the observation value of the sample information. The loss and risk functions are based on the following hypotheses.

Hypothesis 4. The density function of population transfer rate in prior distribution $\pi(\boldsymbol{\theta})$ is known, and the sample information related to $\boldsymbol{\theta}$ can be continuously observed and obtained in cycle $T$. The observation can be conducted numerous times in cycle $T$. The comprehensive observation value is obtained.

The Bayes decision method comprehensively uses the prior information of parameters and sample information to make a decision. The density function of disaster information in prior distribution $\pi(\boldsymbol{\theta})$ can be derived, where $\boldsymbol{\theta}$ is a multidimensional random variable, based on the disaster databases from government departments (e.g., the National Climatic Data Center of the National Oceanic and Atmospheric Administration, the National Geophysical Data Center, and the National Oceanographic Data Center).

The population transfer rate information observation is expressed by $\mathbf{X}^{1}, \mathbf{X}^{2}, \ldots \mathbf{X}^{k}=\left(X_{1}^{k}, X_{2}^{k}, \ldots\right)$ is the sample observed in stage $k$, and $\mathbf{x}^{k}$ refers to the sample information. The condition density function is $f_{k}\left(\mathbf{x}^{k} \mid \boldsymbol{\theta}^{k}\right)$, which is defined in $\mathscr{X}^{k}=\mathscr{X}_{1} \times \mathscr{X}_{2} \times \cdots \times \mathscr{X}_{k}$. The posterior probability density while $\boldsymbol{\theta}^{k}$ is in stage $k$ is $\pi_{k}^{*}\left(\boldsymbol{\theta}^{k} \mid \mathbf{x}^{k}\right) \cdot \boldsymbol{\delta}\left(\mathbf{x}^{k}\right)=\boldsymbol{\delta}\left(x_{1}^{k}, x_{2}^{k}, \ldots\right)$ is the allocation plan for all disaster points.

Hypothesis 5. A difference exists between the emergency supply allocation and actual demand, causing a decrease in the utility of the limited material, which is defined as the firstaid medicine mismatch error losses (MMELs).

A difference always exists between first-aid medicine allocation and actual demand because of the incompleteness of information. Excess or deficiency will reduce the effectiveness 
of drug allocation. The effectiveness of drug allocation will be significant if the allocation error is high. If the demand for resources is set to $d(\theta)$ and the supply is $s$, the first-aid MMEL function can be expressed as follows:

$$
L^{\mathrm{FDMEL}}(\theta, \delta)=(|d(\theta)-s|)^{\beta}, \quad \beta \in R .
$$

4.2. Fair Allocation Principle Based on Demand Information Updating. Equation (11) indicates that the first-aid MMEL function in demand point $j$ in $t$ is

$$
L_{j t}^{\mathrm{FMMEL}}\left(\theta_{j t}, \delta_{j t}\right)=\left(\left|d\left(\theta_{j t}\right)-s_{i j t}\right|\right)^{\beta}, \quad \beta \in R .
$$

In $t$, the first-aid MMEL function of all demand points is

$$
L^{\mathrm{FMMEL}}(\boldsymbol{\theta}, \boldsymbol{\delta})=\left(\left|\sum_{j=1}^{n} d\left(\theta_{j t}\right)-\sum_{i=1}^{m} s_{i t}\right|\right)^{\beta}, \quad \beta \in R .
$$

Equations (12) and (13) do not consider the observation sample information $X^{k}$. Consequently, the effectiveness of decision is affected and the updated data cannot be used. According to the formula of Bayes risk defined in literature (Berger, 1980), the Bayes risk function of first-aid MMEL in demand point $j$ in $t$ is defined as follows:

$$
r_{j t}^{\mathrm{FMMEL}}\left(\pi^{*}, \delta_{j t}\right)=E^{\pi^{*}} E_{\theta_{j t}}^{x_{j t}}\left[L_{j t}^{\mathrm{FMMEL}}\left(\theta_{j t}, \delta_{j t}\right)\right] .
$$

In $t$, the Bayes risk function of first-aid MMEL in all demand points is

$$
r^{\mathrm{FMMEL}}\left(\pi^{*}, \boldsymbol{\delta}\right)=E^{\pi^{*}} E_{\theta_{j t}}^{x_{j t}}\left[L^{\mathrm{FMMEL}}(\boldsymbol{\theta}, \boldsymbol{\delta})\right] .
$$

Definition 6. The fair allocation principle under demand information updating ensures that the Bayes risks of first-aid MMEL in all disaster points are the same; that is,

$$
r_{j t}^{\mathrm{FMMEL}}\left(\pi^{*}, \delta_{j t}\right)=\frac{1}{n} \cdot r^{\mathrm{FMMEL}}\left(\pi^{*}, \boldsymbol{\delta}\right) .
$$

4.3. Decision Model under Demand Information Updating. The optimal decision scheme can be obtained by minimizing the Bayes risk. Based on literature (Berger, 1980), when the posterior distribution is $\pi(\theta \mid x)$, the posterior Bayes expected loss of action $a$ is

$$
\rho(\pi(\theta \mid x), a)=\int_{\Theta} L(\theta, a) d F^{\pi(\theta \mid x)}(\theta) .
$$

Theorem 7 (Berger, 1980). The posterior Bayes action obtained after minimizing (17) is equal to the Bayes action obtained after minimizing

$$
\int_{\Theta} L(\theta, a) f(x \mid \theta) d F^{\pi}(\theta) .
$$

Theorem 8 (Berger, 1980). When $\delta$ is a nonrandomized estimator,

$$
r(\pi, \theta)=\int_{\{x: m(x)>0\}} \rho(\pi(\theta \mid x), \delta(x)) d F^{m}(x) .
$$

The integral sign in Theorems 7 and 8 shows that when random variables are continuous, they are calculated by an integer; when random variables are discrete, they are calculated by a summation formula. Theorems 7 and 8 are applied to (16) to simplify its calculation.

On the basis of the preceding discussion, the equilibrium decision model without information updating can be improved. The model discussed in this paragraph is the equilibrium decision model under demand information updating. Thus, the target function is (6), with unchanged constraints (8), (9), and (10). The related improvement is mainly for constraint (7), and the specific improvement is as follows:

$$
\begin{aligned}
& \int_{\Delta} \int_{\Theta} L_{j t}^{\mathrm{FMMEL}}\left(\theta_{j t}, \delta_{j t}\right) f_{t}\left(x_{j t} \mid\right. \\
& \left.\theta_{j t}\right) d F^{\pi}\left(\theta_{j t}\right) d F^{u}\left(x_{j t}\right) \\
& \left(\Delta=\left\{x_{j t}: u\left(x_{j t}\right)>o\right\}\right), \\
& =\int_{\Delta} \int_{\Theta} L^{\mathrm{FMMEL}}(\boldsymbol{\theta}, \boldsymbol{\delta}) f_{t}(\mathbf{x} \mid \boldsymbol{\theta}) d F^{\pi}(\boldsymbol{\theta}) d F^{u}(\mathbf{x}) \\
& (\Delta=\{\mathbf{x}: u(\mathbf{x})>0\}) .
\end{aligned}
$$

Meanwhile, the Bayes-risk-related calculation formula is added as follows:

$$
\begin{aligned}
u\left(x_{j t}\right) & =\int_{\Theta} f\left(x_{j t} \mid \theta_{j t}\right) d F^{\pi}\left(\theta_{j t}\right), \quad \forall j \in J, \\
h\left(x_{j t}, \theta_{j t}\right) & =\pi\left(\theta_{j t}\right) f\left(x_{j t} \mid \theta_{j t}\right), \quad \forall j \in J, \\
\pi^{*}\left(\theta_{j t} \mid x_{j t}\right) & =\frac{h\left(x_{j t}, \theta_{j t}\right)}{u\left(x_{j t}\right)}, \quad \forall j \in J .
\end{aligned}
$$

Equation (20) refers to the mathematical expression of the fair allocation principle under demand information updating, (21) is the marginal probability density formula, (22) is a joint probability density formula, and (23) is the posterior probability density formula.

\section{Numerical Simulation Analysis}

5.1. Background and Data. A shallow-source great earthquake at magnitude 8.0 suddenly occurred at 2:28:04 PM, China Standard Time, on May 12, 2008, in WC and Beichuan (BC), Sichuan Province. The maximum intensity was 11 . This earthquake was the strongest earthquake in the area with the greatest destruction since the founding of new China. A total of 417 counties, 4,656 towns, and 47,789 villages in Sichuan, Gansu, Shanxi, Chongqing, and other provinces (districts and cities) were affected by the earthquake. Among these areas, 10 disaster areas in the Sichuan Province were seriously affected. The WC earthquake disaster situation is shown in Table 1 . The total casualties in the 10 severe disaster areas are shown in Table 2. In consideration of the significant impact of the WC earthquake and data availability, a simulation analysis of the first-aid medicine allocation in 
TABLE 1: Victims and casualties in the WC earthquake (unit: people, square kilometer).

\begin{tabular}{lcccccc}
\hline Scope & Death & Missing & Injured & Transferred population & Affected population & Affected area \\
\hline Country & 69,181 & 18,498 & 374,171 & $1,510,000$ above & $46,260,000$ & 440,000 \\
Sichuan & 68,669 & 18,498 & 360,352 & $1,460,000$ above & $29,830,000$ & 280,000 \\
\hline
\end{tabular}

Data resources: report on the 2008 Sichuan big earthquake.

TABLE 2: Total casualties and disaster index in 10 severe disaster areas.

\begin{tabular}{lcccccccccc}
\hline Disaster points & WC & BC & MZ & SF & QC & MX & AX & DJY & PW & PZ \\
\hline Population (10 thousand people) & 11.2 & 16.1 & 51.6 & 43.3 & 25.0 & 10.4 & 48.4 & 62.2 & 18.6 & 77.0 \\
Death/missing (people) & 23,871 & 20,047 & 11,380 & 6132 & 4819 & 4088 & 3295 & 3388 & 6565 & 1131 \\
Injured (people) & 34,583 & 9693 & 36,468 & 31,990 & 15,453 & 8183 & 13,476 & 4388 & 32,145 & 5770 \\
Average intensity & 8.89 & 9.16 & 9.14 & 8.68 & 8.74 & 7.91 & 8.89 & 9.13 & 8.15 & 8.53 \\
Comprehensive disaster index & 0.8675 & 0.7050 & 0.6612 & 0.5953 & 0.5146 & 0.5107 & 0.4993 & 0.4910 & 0.4424 & 0.4333 \\
\hline
\end{tabular}

Data resources: Ministry of Civil Affairs, Ministry of Finance, Ministry of Science and Technology, China Earthquake Administration, and China National Commission for Disaster Reduction.

the 10 severe disaster areas was conducted by using the earthquake first-aid medicine equilibrium decision model under an uncertain environment. Figure 1 shows that the rescue supplies across the country were first distributed to these four points and then sent to WC, BC, Mianzhu (MZ), Shifang (SF), Qingchuan (QC), Maoxian (MX), Anxian (AX), Dujiangyan (DJY), Pingwu (PW), Pengzhou (PZ), and other disaster areas with Chengdu (CD), Deyang (DY), Mianyang (MY), and Guangyuan (GY) as the rescue points.

In the most severely affected areas in the WC earthquake, the main highway transportation tools participating in material distribution were various trucks, and the main air tools were helicopters (Black Hawk, Zhi 8, Mi 17 series, Mi 26, etc.). In consideration of the importance of first-aid medicine, a helicopter is used for transport in the simulation analysis. The driving distance among all nodes in the distribution network and the driving time are shown in Table 3. The medicine supplies in all supply points are shown in Table 4.

5.2. Equilibrium Time Point between the Risks of Decision Error and Delay. As time passes, the forecast accuracy increases, the risk of decision error decreases, and the risk of decision delay increases. Therefore, the calculation formula of forecast accuracy $p(t)$ adopted in this study is as follows:

$$
p(t)=\frac{1}{\left(T_{0}-t+1\right)}
$$

The calculation formula of the risk of decision error is

$$
R_{t}^{\text {Error }}=1-p(t)=1-\frac{1}{\left(T_{0}-t+1\right)} .
$$

The calculation formula of the risk of decision delay is

$$
R_{t}^{\text {Delay }}=R(t)=\frac{t}{T_{0}}
$$

where $0<t<T_{0}$ and $T_{0}$ refers to the length of decision cycle, which can be set to $24 \mathrm{~h}$. The calculation formulas of forecast accuracy (24), the risk function of decision error
(25), and the risk of decision delay (26) can be modified based on the specific conditions. According to the above formulas, when decision time $t \in[14.1903,22.3909]$ can be obtained as $\alpha=0.1$, the risk of decision error balances the risk of decision delay. When decision time $t=19.5756$, the difference between the risks of decision error and delay is 0 . The function graphs of the risk of decision error, risk of decision delay, and the balance between them are shown in Figure 2.

5.3. Distribution of Random Variables for Demand and Setting of Other Calculation Formulas. The calculation formula of the demand in all disaster areas is

$$
d_{j t}=d\left(\theta_{j t}\right)=N_{j} \cdot \gamma_{j} \cdot \lambda \cdot \theta_{j t}
$$

where $N_{j}$ refers to the population in disaster point $j, \gamma_{j}$ refers to the casualty ratio in disaster point $j$, and $\lambda$ refers to the demand per casualty for a certain drug.

The historical seismic data published by the China National Committee for Disaster Reduction and the Ministry of Science and Technology can be used as reference to check if the various status sets of population transfer rate $\Theta=\{\boldsymbol{\theta}\}$ obey the distribution by population transfer rate and road destructive rate in the 10 severe disaster areas and 36 severe disaster counties in the WC earthquake. The data are shown as follows.

Population Transfer Rate. 1.0223, 0.8625, 0.9029, 0.8529, $0.9811,1.2824,0.9720,0.7174,0.7767,1.0008,0.5150,0.8476$, $0.5229,0.4261,0.7970,0.5351,0.6398,0.8000,0.1151,0.2666$, $0.5037,0.4656,0.5241,0.5811,0.3169,0.3687,0.1085,0.1375$, $0.2570,0.663,0.3151,0.2138,0.9789,0.5754,1.2792,0.5511$, $0.4385,0.8119,0.3401,0.1708,0.3565,0.9075,0.5313,0.5220$, $0.3713,0.3350,-,-$.

On the basis of the above data, SPSS is used to make a statistical analysis, as shown in Figure 3 and Table 5. 
TABLE 3: Driving distance among all nodes in the distribution network and the driving time.

\begin{tabular}{lcccccccccc}
\hline & WC & BC & MZ & SF & QC & MX & AX & DJY & PW & PZ \\
\hline CD & $100 / 35$ & $135 / 45$ & $76 / 28$ & $53 / 21$ & $240 / 77$ & $116 / 40$ & $108 / 37$ & $57 / 22$ & $200 / 65$ & $40 / 17$ \\
DY & $85 / 30$ & $78 / 28$ & $30 / 14$ & $22 / 12$ & $180 / 59$ & $80 / 29$ & $48 / 20$ & $76 / 28$ & $143 / 48$ & $45 / 19$ \\
MY & $103 / 36$ & $45 / 19$ & $48 / 20$ & $62 / 24$ & $135 / 46$ & $82 / 30$ & $13 / 10$ & $115 / 40$ & $105 / 37$ & $86 / 31$ \\
GY & $238 / 76$ & $146 / 49$ & $198 / 65$ & $215 / 70$ & $60 / 23$ & $205 / 67$ & $156 / 56$ & $265 / 85$ & $124 / 42$ & $240 / 77$ \\
\hline
\end{tabular}

Note: the unit is $\mathrm{km} /$ minute; the flying speed is $200 \mathrm{~km} / \mathrm{h}$.
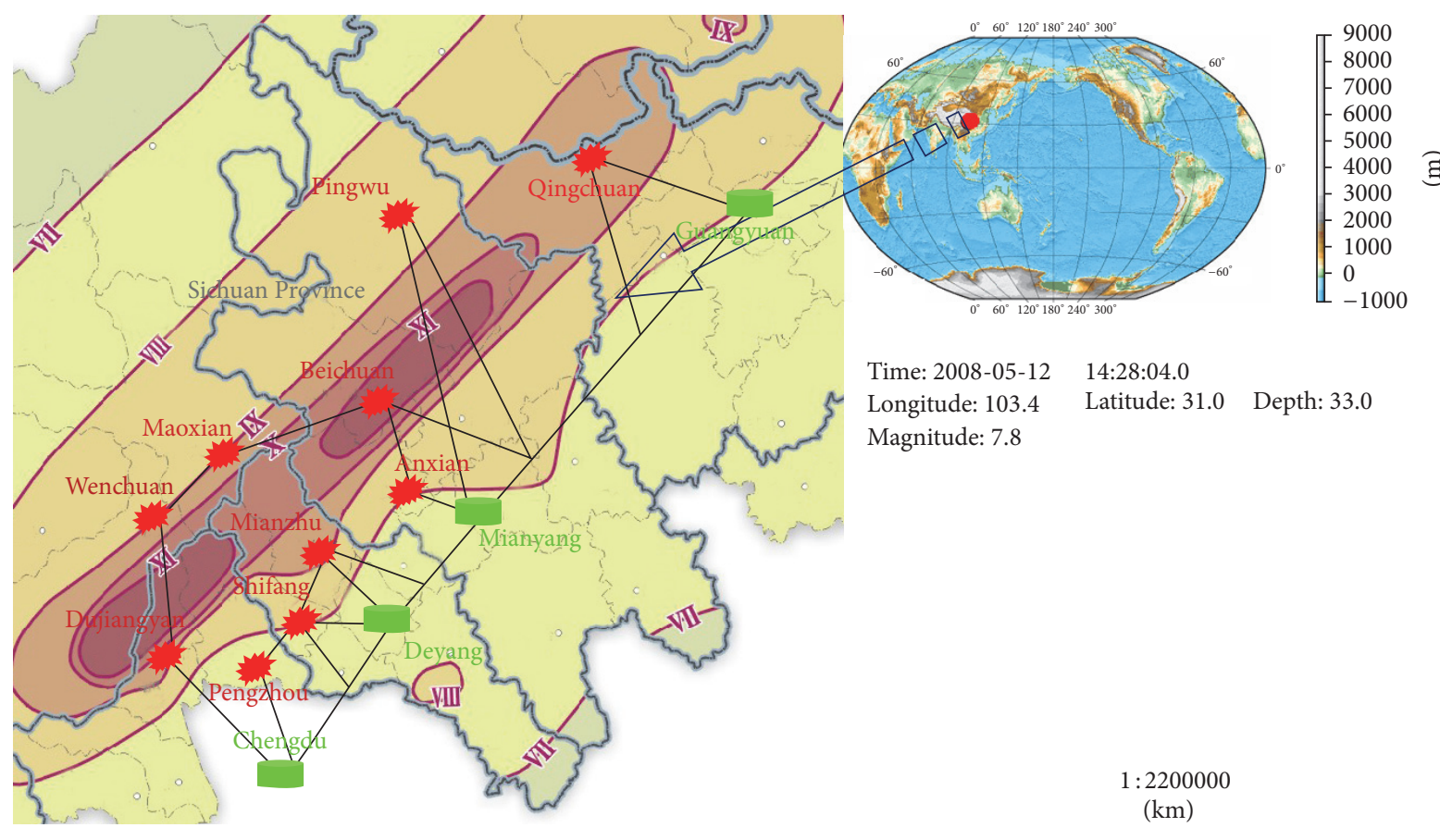

Time: 2008-05-12 14:28:04.0

Longitude: 103.4 Latitude: 31.0 Depth: 33.0

Magnitude: 7.8

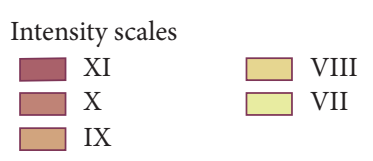

Logistics network
Most severely affected areas
Allocating centers

FIGURE 1: Distribution of severe disaster areas and main rescue points in the WC earthquake. Data resources: China Seismological Bureau, National Wenchuan Earthquake Reconstruction Planning Group, Google Earth.

TABLE 4: Driving distance among all nodes in the distribution network and the driving time.

\begin{tabular}{lcccc}
\hline Supply points & CD & DY & MY & GY \\
Quantity of drug A & 35000 & 14000 & 13999 & 6999 \\
\hline
\end{tabular}

The examination result is shown in Table 6. The population transfer rate $\theta_{j t}$ obeys the following distribution in $[0,1]$ :

$$
\theta_{j t} \cdot N\left(\mu, \sigma^{2}\right), \quad \forall j \in J, t \in T .
$$

Table 7 shows the statistics of sample data under the assumption that the population transfer rate of this earthquake in all severe disaster areas is sampled.

5.4. Calculation Result. On the basis of the above settings, let $\gamma_{j}=0.02(j=1,2, \ldots, 10), \lambda=2, \beta=2$; then
TABLE 5: Statistical table of population transfer rate.

\begin{tabular}{lc}
\hline Description & Population transfer rate \\
\hline$N$ & \\
$\quad$ Valid & 46 \\
$\quad$ Missing & 0 \\
Mean & .596971739 \\
Std. deviation & .3013077317 \\
Skewness & .362 \\
Std. error of skewness & .350 \\
Kurtosis & -.546 \\
Std. error of kurtosis & .688 \\
\hline
\end{tabular}

the equilibrium decision method of the first-aid medicine allocation without information updating can be used to 
TABLE 6: One-sample Kolmogorov-Smirnov test result.

\begin{tabular}{lc}
\hline Description & Population transfer rate \\
\hline$N$ & 46 \\
Normal parameters $(a, b)$ & \\
$\quad$ Mean & .596971739 \\
$\quad$ Std. deviation & .3013077317 \\
Most extreme differences & \\
Absolute & .108 \\
Positive & .108 \\
Negative & -.073 \\
Kolmogorov-Smirnov $Z$ & .732 \\
Asymp. sig. (2-tailed) & .657 \\
\hline
\end{tabular}

$a$ : the test distribution is normal.

$b$ : calculated from data.

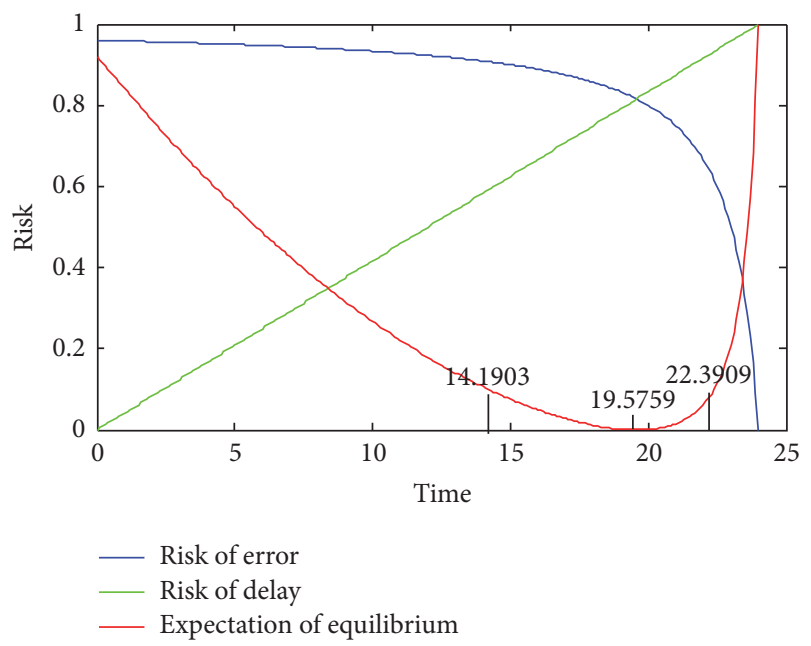

FIGURE 2: Function graphs of risk of decision error, risk of decision delay, and the balance between them.

obtain the decision-making time $t \in[14.1903,22.3909]$. The detailed allocation scheme is shown in Table 8 .

The equilibrium decision method of the first-aid medicine allocation under demand information updating is used to obtain the decision-making time $t \in[14.1903$, 22.3909]. The detailed allocation scheme is shown in Table 9.

Meanwhile, we can compare the population, allocation amount without information updating, allocation amount, and population multiplied by the comprehensive disaster index in each disaster area of the total. The comparison results are shown in Figure 4. The allocation amount without information updating fits the population in each disaster area well. By contrast, the allocation amount with information updating fits the population multiplied by the comprehensive disaster index in each disaster area better. Therefore, the decision method with demand information updating better fits the real disaster situation based on historical and sample information.

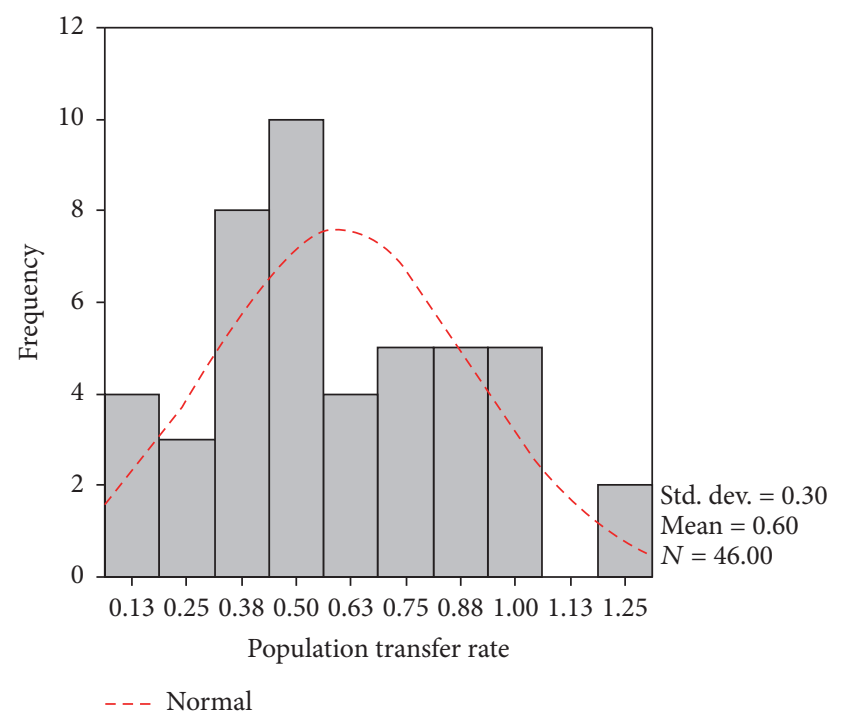

FIGURE 3: Frequency histogram of population transfer rate.

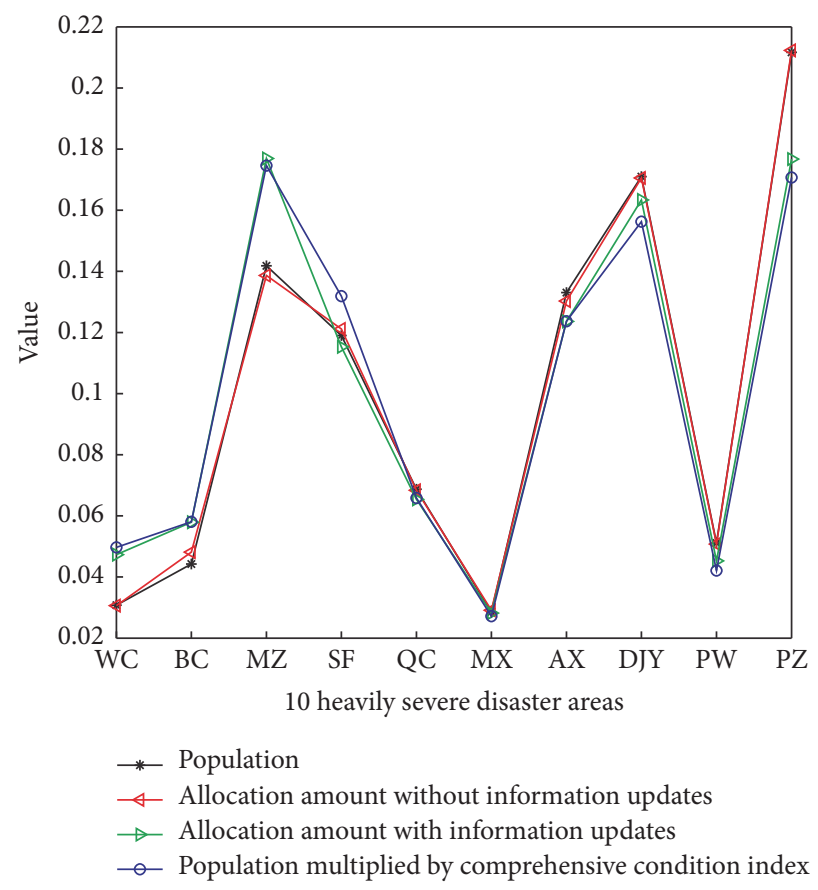

FIGURE 4: Comparison of the population and allocation amount.

\section{Conclusion}

Unlike the allocation of general first-aid supplies, earthquake first-aid medicine allocation is specific. The transport constraint is relatively small and the scientific nature of the allocation scheme must be high. In this study, according to the change in the supply and demand for first-aid drugs, the balance between the risks of decision error and delay reflects that the square of their difference is not beyond the constraint on a certain expected value. The expected demand satisfaction rates of all disaster areas should be the same, and the Bayes risk of first-aid medicine allocation 
TABLE 7: Statistical value of population transfer rate sample data in severe disaster areas.

\begin{tabular}{lccccccccccc}
\hline Demand points & WC & BC & MZ & SF & QC & MX & AX & DJY & PW & PZ & Total \\
\hline Mean value & 0.61 & 0.53 & 0.45 & 0.47 & 0.51 & 0.48 & 0.46 & 0.57 & 0.53 & 0.41 & 0.502 \\
Variance & 0.09 & 0.10 & 0.12 & 0.09 & 0.11 & 0.09 & 0.12 & 0.10 & 0.11 & 0.09 & 0.10 \\
\hline
\end{tabular}

TABLE 8: Allocation scheme of drug A.

\begin{tabular}{lcccccccccc}
\hline & WC & BC & MZ & SF & QC & MX & AX & DJY & PW & PZ \\
\hline CD & 2145 & 0 & 0 & 6055 & 0 & 0 & 0 & 11939 & 14861 \\
DY & 0 & 0 & 9704 & 2426 & & 1870 & 0 & 0 & 0 \\
MY & 0 & 3368 & 0 & 0 & 0 & 169 & 9120 & 0 & 1342 & 0 \\
GY & 0 & 0 & 0 & 0 & 4786 & 0 & 0 & 0 & 2213 & 0 \\
\hline Total & 2145 & 3368 & 9704 & 8481 & 4786 & 2039 & 9120 & 11939 & 3555 & 14861 \\
\hline
\end{tabular}

loss in all demand points should be equal to the total Bayes risk to achieve fair allocation to all disaster areas. This study also introduces a set of equilibrium decision methods for earthquake first-aid medicine allocation based on information updating to minimize the total transport time loss. Finally, the simulation analysis is applied to a certain drug allocation in the WC earthquake to verify the effectiveness of the constructed method. This study can provide enlightenment on the following management decisions for emergency decision makers:

(1) In earthquake first-aid medicine allocation decision, the relation between decision-making time and risk is important. A decision maker must realize the balance between the risks of decision error and delay to realize the maximization of allocation efficiency.

(2) The decision-making information in an earthquake disaster environment is incomplete. Bayes analysis is an effective method for realizing the integration of the related history and sample information.

However, the method proposed in this study has numerous limitations. Future studies on earthquake firstaid medicine allocation can comprehensively consider the changes in supply and demand, the balance between the risks of decision error and delay under information updating, time window constraint, and comprehensive allocation in a timespace network.

\section{Notations}

(i) Symbols

Set

I: Supply point set, $i \in I$

$J$ : Disaster point set, $j \in J$

\section{Stochastic Variables}

$\boldsymbol{\theta}$ : Population transfer rate

$\theta_{j t}$ : Population transfer rate in disaster point $j$ in $t$

\section{Variables}

$d_{j t}: \quad$ Requirement of point $j$ in $t$

$r_{t}$ : Demand prediction accuracy in $t$

$R_{t}^{\text {Error }}$ : Loss of error decision in $t$

$R_{t}^{\text {Delay }}$ : Loss of delay decision in $t$

$L_{t}^{T}$ : Loss of transportation time of the decision-making program in $t$

$u_{j t}$ : Expected demand satisfaction rate of point $j$ in $t$

\section{Decision Variables}

$t$ : Decision time

$s_{i j t}$ : Requirement of point $j$ in $t$ from supply point $i$

Parameters

$t_{i j}^{0}$ : Transport time in point $j$ from supply point $i$

$S_{i t}$ : Requirement in point $i$ in $t$.

(ii) New Symbols

Stochastic Variables

$\mathbf{X}$ : Independent observation value of $\boldsymbol{\theta}$

$\mathbf{x}$ : Specific realization value of $\mathbf{X}$

a: Specific allocation plan of rescue drugs.

Set

$\Theta: \quad$ All possible $\boldsymbol{\theta}$ set

$\mathscr{X}$ : All possible $\mathbf{X}$ set

$\mathscr{A}:$ All possible a set

$N^{+}$: All positive integer set.

\section{Functions and Equations}

$\pi(\boldsymbol{\theta})$ : $\quad$ Prior probability density function of $\boldsymbol{\theta}$

$u(\mathbf{x})$ : Edge probability density function of $\mathbf{x}$

$h(x, \boldsymbol{\theta})$ : Joint probability density function of $\mathbf{x}$ and $\boldsymbol{\theta}$

$f(\mathbf{x} \mid \boldsymbol{\theta})$ : Condition probability density function of

$\mathbf{x}$ 
TABLE 9: Allocation scheme of drug A.

\begin{tabular}{lcccccccccc}
\hline & WC & BC & MZ & SF & QC & MX & AX & DJY & PW & PZ \\
\hline CD & 3311 & 0 & 0 & 7884 & 0 & 0 & 0 & 11435 & 12370 \\
DY & 0 & 0 & 12384 & 187 & & 1429 & 0 & 0 & 0 & 0 \\
MY & 0 & 4054 & 0 & 0 & 0 & 545 & 8653 & 0 & 747 & 0 \\
GY & 0 & 0 & 0 & 0 & 4578 & 0 & 0 & 0 & 2421 & 0 \\
\hline Total & 3311 & 4054 & 12384 & 8071 & 4578 & 1974 & 8653 & 11435 & 3168 & 12370 \\
\hline
\end{tabular}

$\pi^{*}(\boldsymbol{\theta} \mid \mathbf{x})$ : Posterior probability density function of $\boldsymbol{\theta}$

$F^{\pi}(\boldsymbol{\theta})$ : Cumulative distribution function of $\boldsymbol{\theta}$

$r_{t}^{\text {FMMEL }}$ : Bayesian risk of loss of rescue drug allocation error in $t$

$r_{j t}^{\mathrm{FMMEL}}$ : Bayesian risk of loss of rescue drug allocation error in disaster point $j$ in $t$

$F^{u}(\mathbf{x})$ : Marginal cumulative distribution function of $\mathbf{x}$

$\boldsymbol{\delta}(\mathbf{x}): \quad$ Decision in space $\mathscr{X} \boldsymbol{\delta}(\mathbf{x})$ is a plan a $\in \mathscr{A}$ under the condition of $\mathbf{X}=\mathbf{x}$.

\section{Competing Interests}

The authors declare that they have no competing interests.

\section{Acknowledgments}

This work is supported by the National Natural Science Foundation of China (Grant no. 71601146), Humanities and Social Sciences Foundation of Ministry of Education of China (Grant no. 15YJCZH211), Zhejiang Provincial Philosophy and Social Science Program of China (Grant no. 16ZJQN025YB), Zhejiang Provincial Natural Science Foundation of China (Grant no. LQ16G010005), and Zhejiang Provincial College Major Program of Humanities and Social Science of China (Grant no. 2014QN006).

\section{References}

[1] S. H. Jian and Y. Y. Li, "Significance of index of thetotal medical rescuer esponse in Ya an earthquake medical rescue," Journal of Traumatic Surgery, vol. 17, no. 1, pp. 62-63, 2015.

[2] T. K. Becker, G. A. Jacquet, R. Marsh et al., "Global emergency medicine: a review of the literature from 2013," Academic Emergency Medicine, vol. 21, no. 7, pp. 810-817, 2014.

[3] A. Hairapetian, A. Alexanian, M.-C. Férir et al., "Drug supply in the aftermath of the 1988 Armenian earthquake," The Lancet, vol. 335, no. 8702, pp. 1388-1390, 1990.

[4] H.-T. Xiao, Z. Liao, L. Chen, and R.-S. Tong, "The donation drug of Wenchuan earthquake-focus on Chinese national," HealthMED, vol. 4, no. 2, pp. 356-359, 2010.

[5] J. Mori, K. Hasui, T. Tanimoto, T. Matsumura, and M. Kami, "Drug shortages after the Eastern Japan Earthquake: experiences in a tertiary referral center," Drug Information Journal, vol. 46, no. 5, pp. 607-610, 2012.

[6] M. S. Sever, R. Vanholder, and RDRTF of ISN Work Group on Recommendations for the Management of Crush Victims in Mass Disasters, "Recommendation for the management of crush victims in mass disasters," Nephrology Dialysis Transplantation, vol. 27, supplement 1, pp. 1-67, 2012.

[7] R. Kazancioglu, B. Pinarbasi, B. A. Esen, A. Turkmen, and M. S. Sever, "The need for blood products in patients with crush syndrome," American Journal of Disaster Medicine, vol. 5, no. 5, pp. 295-301, 2010.

[8] D. Kudo, H. Furukawa, A. Nakagawa et al., "Resources for business continuity in disaster-based hospitals in the Great East Japan Earthquake: survey of miyagi prefecture disaster base hospitals and the prefectural disaster medicine headquarters," Disaster Medicine and Public Health Preparedness, vol. 7, no. 5, pp. 461-466, 2013.

[9] J.-B. Sheu, "Post-disaster relief-service centralized logistics distribution with survivor resilience maximization," Transportation Research Part B: Methodological, vol. 68, pp. 288-314, 2014.

[10] A. Haghani, S. Yan, and Y. L. Shih, "Optimal scheduling of emergency roadway repair and subsequent relief distribution," Computers \& Operations Research, vol. 36, no. 6, pp. 2049-2065, 2009.

[11] L. Özdamar and W. Yi, "Greedy neighborhood search for disaster relief and evacuation logistics," IEEE Intelligent Systems, vol. 23, no. 1, pp. 14-23, 2008.

[12] F.-S. Chang, J.-S. Wu, C.-N. Lee, and H.-C. Shen, "Greedysearch-based multi-objective genetic algorithm for emergency logistics scheduling," Expert Systems with Applications, vol. 41, no. 6, pp. 2947-2956, 2014.

[13] X. G. Zhang, Z. L. Zhang, Y. J. Zhang, D. Wei, and Y. Deng, "Route selection for emergency logistics management: a bioinspired algorithm," Safety Science, vol. 54, no. 10, pp. 87-91, 2013.

[14] Y. Yuan and D. Wang, "Path selection model and algorithm for emergency logistics management," Computers \& Industrial Engineering, vol. 56, no. 3, pp. 1081-1094, 2009.

[15] J. O. Berger, Statistical Decision Theory and Bayesian Analysis, Springer, New York, NY, USA, 2nd edition, 1980. 


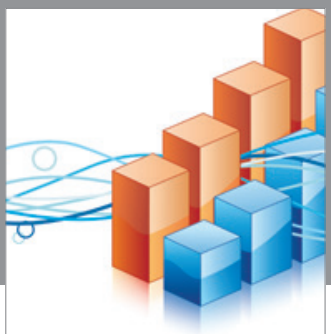

Advances in

Operations Research

vatem alat4

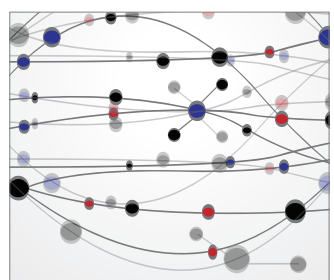

\section{The Scientific} World Journal
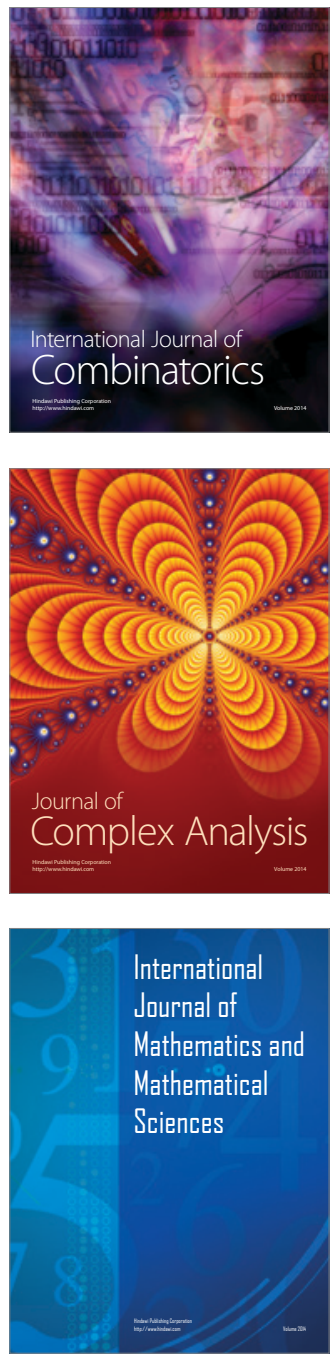
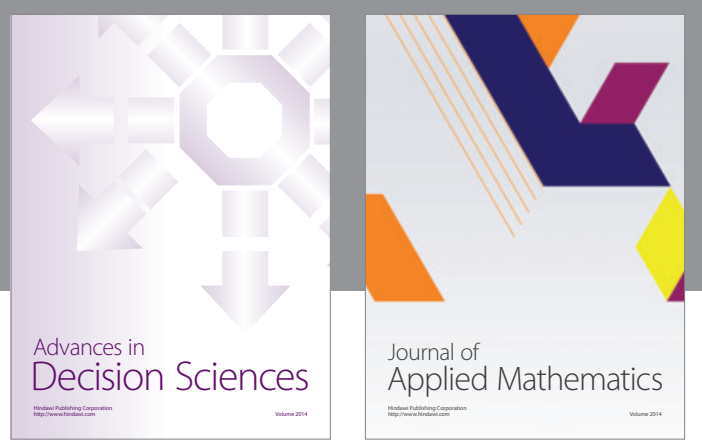

Algebra

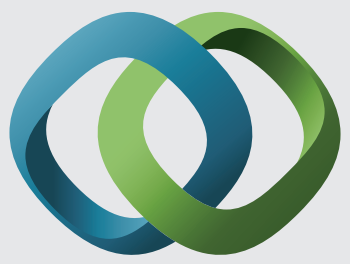

\section{Hindawi}

Submit your manuscripts at

https://www.hindawi.com
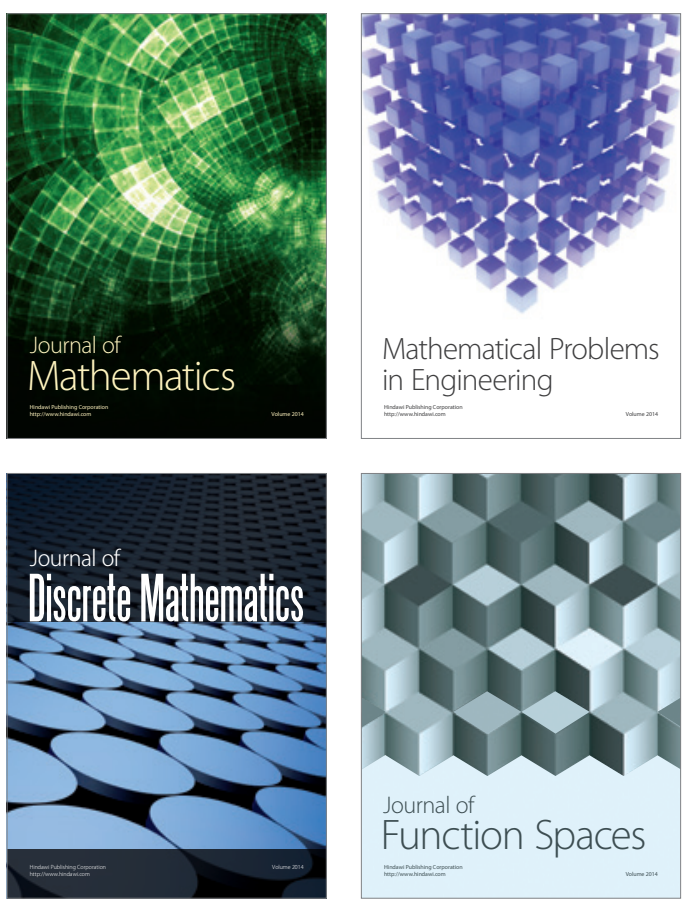

Mathematical Problems in Engineering
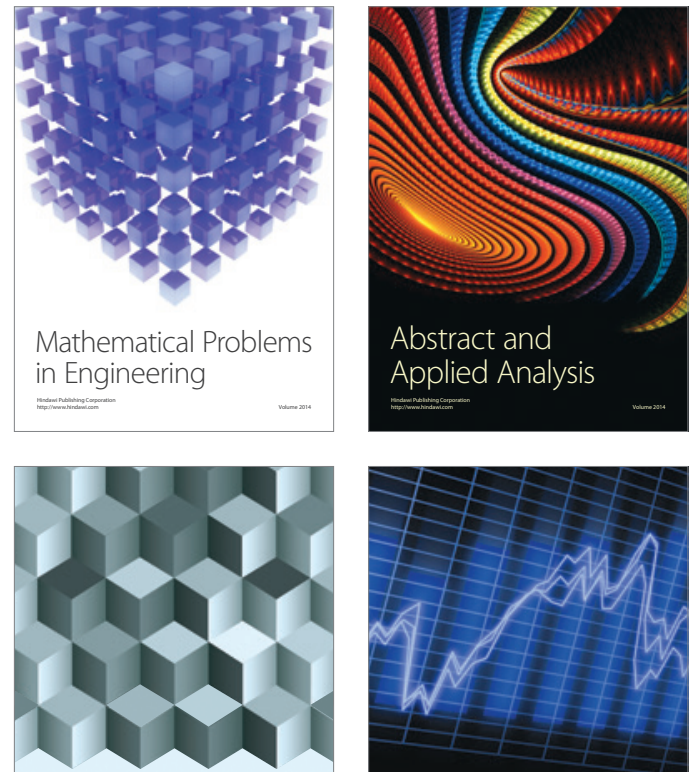

Journal of

Function Spaces

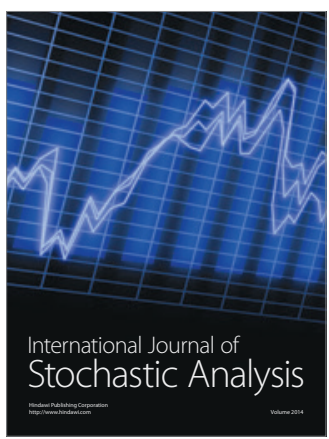

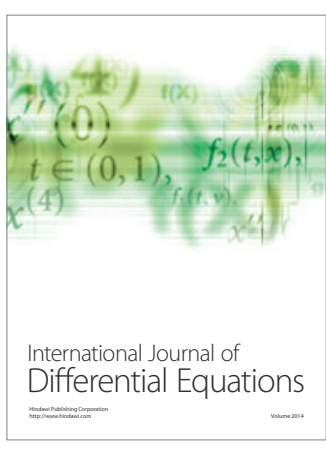
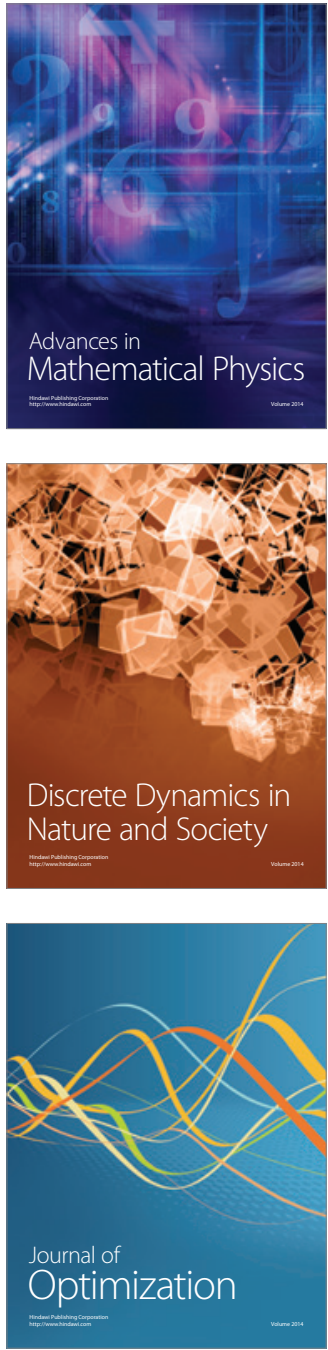